\title{
An approach for manufacturing process representation in product lifecycle management
}

\author{
Navid Shariatzadeh ${ }^{1, a}$, Gunilla.Sivard ${ }^{1, b}$ Lars Lindberg $^{1, c}$ \\ ${ }^{1}$ Brinellvägen 68, 10044, Stockholm, Sweden \\ ansh@kth.se, ${ }^{\mathrm{a}}$ gunilla.sivard@iip.kth.se, ${ }^{\mathrm{C} l l i @ i i p . k t h . s e ~}$
}

Keywords: Manufacturing flow, Information management, Ontology

\begin{abstract}
Current Product Lifecycle Management systems (PLM) have concentrated on product design, not on manufacturing engineering with its development of e.g. Material flows and layouts. This paper proposes an approach to describe how to represent the main required manufacturing process data using ontologies together with generic data standards. This approach makes it possible to develop translations between different software, and also providing users with the meaning of different concepts. It contributes to an efficient management of manufacturing information, with a focus on the material flow information as used in Discrete Event Simulation - DES.
\end{abstract}

\section{Introduction}

The multi-disciplinary and knowledge intensive nature of DES models requires collecting and structuring data and information about the manufacturing process and product routing as well as manufacturing facts and rules. However today this information is normally not integrated in one place, but is heterogeneous and stored in different Computer Aided Design (CAD) and Computer Aided Manufacturing (CAM) applications in the factory plant. The lack of interoperability among these systems results in inefficiency of building DES models in terms of cost and time. Therefore; incorporating a data structure that represents manufacturing systems and can be integrated with other types of information such as geometry, e.g. for sizes of products or distances of transports, is of vital interest. Moreover since DES modeling is a multi-disciplinary task there is a need to agree on information models that facilitates communication and sharing of information between different systems and their users.

\section{Related work}

Much research has already been conducted on information modeling required for manufacturing processes, DES, online simulation, NC data and interlocking [1-2], However, little has been investigated concerning the connection between information and DES models and integrating this information with other type of data. Furthermore; More than 20 methods, including languages, methodologies, tools and standards, have been reported to specify processes of different domains [3]. Nevertheless; few process modeling methods are able to completely represent the complexities and varieties of multiple manufacturing processes of a product.

\section{Required information for material flow and process control}

Required information for material flow representation and process control can be categorized as below:

Product: Product breakdown structure and its geometry data are used for DES. For instance; the diameter of a cylinder is necessary to be able to identify the required capacity of a conveyor which is used in a DES model.

Layout: layout information such as general definitions of the boundary, placement and spatial relations of the manufacturing entities. 
Maintenance: manufacturing resources availability information such as uptime, downtimes.

Process-plan: description of manufacturing processes sequence and product routing such as Processing precedence, Alternative sequences, Parallel actions, Synchronization, non-deterministic processes (various probable activity occurrences might happen in real time according to real time conditions).

Enterprise resource planning: information about orders, production scheduling and production strategy.

Another important aspect when describing the behavior of a resource or a manufacturing process is time. Time consideration can be made by representing requirements and constraints and conditions under which the process is able to perform its task in real time. For instance if a process needs a resource which is busy at a specific time point then it can use another resource or another process can be executed (change the processes ordering).Synthesis of states and events are described by means of logical operands including Negation, And, Or, Implication, Reverse implication.

\section{Proposed approach}

The approach is to use the PSL ontology (Process Specification Language) as a reference for manufacturing processes specification and map it to the STEP standard (Standard for the Exchange of Product model data) for the purpose of increasing the level of interoperability among systems and users. Figure 1 illustrates a schematic demonstration of the proposed approach to represent manufacturing processes for the purpose of DES and process control. The approach basically takes advantage of the PSL ontology to define manufacturing processes concepts, their real time executions, facts, and constraints; and tries to map them to information standard models such as STEP AP239 (PLCS Product Lifecycle Support)).

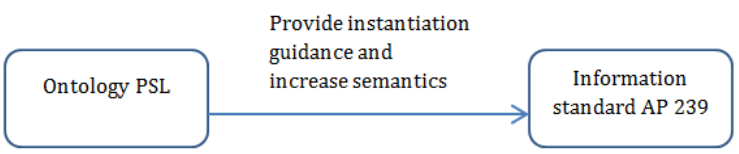

Figure 1. Schematic shape of proposed approach

As it was discussed before one substantial constituent of the approach is using information standards. Information standards connect the concepts of a manufacturing process domain with events and processes and the physical representation of those concepts in a database or PLM system. Generally it is possible to directly create an information model (defining required information and its structure) based on an ontology for a specific domain. However it is a time consuming and labour intensive task. It requires sufficient experience and interviewing domain experts. This issue is a significant reason of the approach to take advantage of information standards particularly ISO 10303 (STEP) Application Protocol 239 (PLCS). This can minimize duplicate efforts needed to develop the information model. ISO 10303 STEP is an international standard that "provides a representation of product information along with the necessary mechanisms and definitions to enable product data to be exchanged".

STEP- AP239 (PLCS) [3] has been developed to represent any product during its life cycle. The applicability of this standard for representing manufacturing system information has been tested in other research [4].

PLCS and other STEP modules have schemas for representation of activity, activity relationship, States, Events that is sufficient for material flow representation. However, PLCS lacks the semantics of the manufacturing process domain that is the description of the logic of the material flow. Therefore our approach deploys the PSL ontology for representing the flow semantics. In this approach PSL is intended to give details and explain the world of manufacturing process, while STEP is used to describe the part of the world whose representation needs to provide users with right data, right version, at right time with the desired level of detail. 
What we mean here by semantics of PSL is the set of concepts, relations, functions, relations and axioms that cover all runtime executions traces of material flow (occurrence tree) and specify which execution traces are allowed under specific conditions. It can disambiguate possible process occurrence in real time.

PSL ontology: The Process Specification Language (PSL) of the National Institute of Standards and Technology (NIST) is a system neutral, standard language for process specification to serve as an Interlingua to integrate multiple process-related applications throughout the manufacturing life cycle [5]. It helps to describe behavior of manufacturing processes from a run time perspective.

The PSL Ontology has a set of theories in the language of first-order logic. The underlying grammar used for PSL is based roughly on the grammar of KIF (Knowledge Interchange Format) [24]. KIF is a formal language based on first-order logic developed for the exchange of knowledge among different computer programs with disparate representations. This also helps for creating inferences engines for reasoning process. The ontology supports interoperability among first-order inference engines that exchange first-order sentences. The PSL ontology includes Formal definition of semantic concepts and their relations for capturing manufacturing processes in real time and supports non deterministic process occurrences.

Required entities in STEP AP 239 PLCS: Figure 2 presents a data model including the main entities and their relations in PLCS for the purpose of manufacturing process representation according to the Module Interpreted Model (MIM) of the standard. The schemas which are used for this purpose include Activity_recording, Product_definition_information, Event, Event assignment, State, State_definition and resource_item. The entity Activity is defined as the identification of the occurrence of an action that has taken place, is taking place, or is expected to take place in the future. Therefore it can be used to represent a manufacturing process in real time. Action resource entity can represent a resource which is used by a process. Events can be used to represent different events based on time to initiate a process. States can be used to represent pre-states and post-states of inputs and outputs of a process or a resource when performing a process. This schema does not include attributes of these entities and detail instantiation specification.

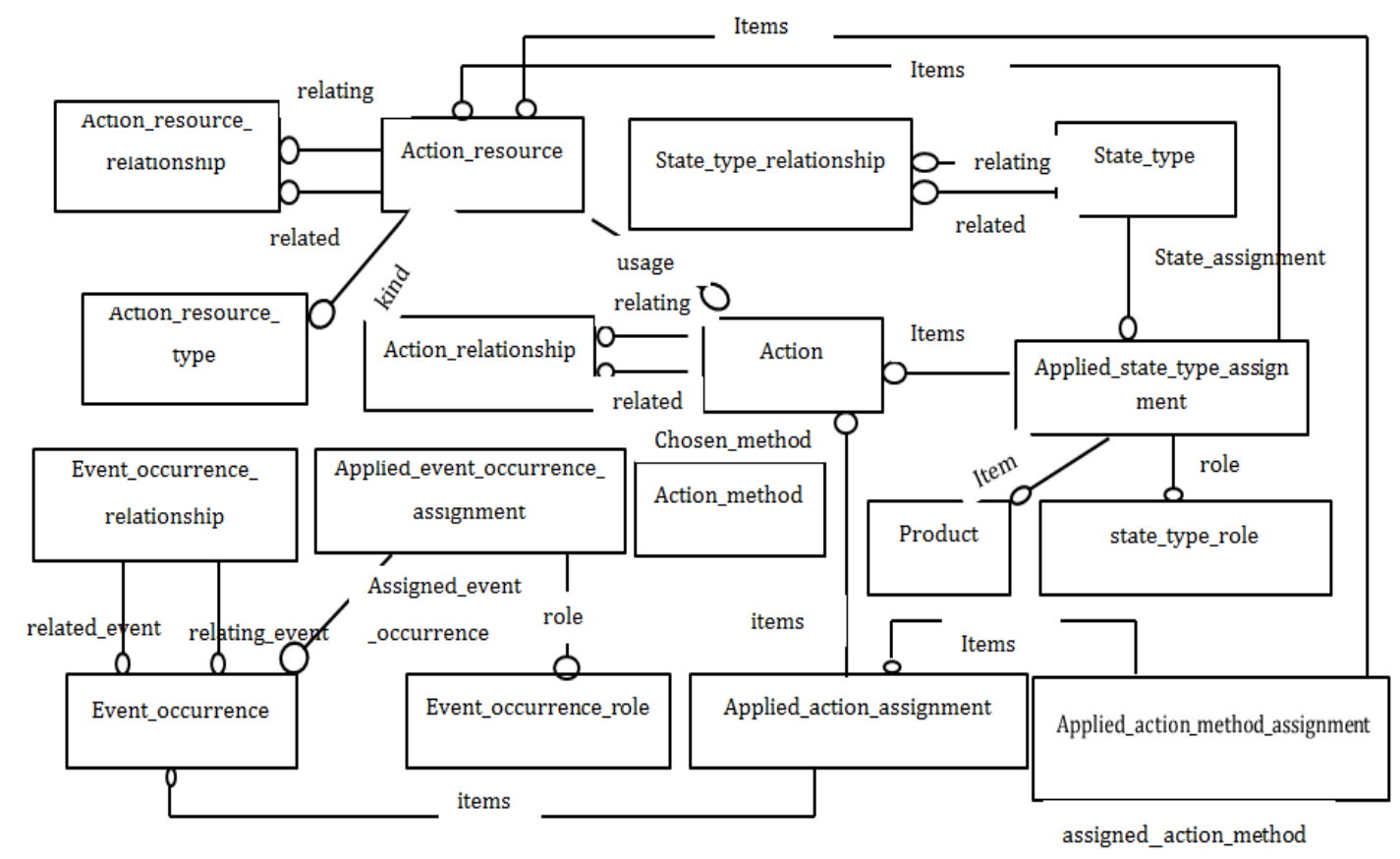

Figure 2. The developed schema for material flow repesntation based on STEP AP 239 


\section{Map PSL to STEP}

Activities and their corresponding occurrences can be mapped to actions and action_relationships in STEP while considering defined relations in PSL based on the type of activities. For example the "next_subocc" relation is a relationship type between two subactivity occurrences which does not allow any occurrence between to sub activity occurrence. While "min_precedes" relation represents that one sub activity occurrence must occur earlier than the other one and other sub activities can occur in between. Activities and their possible occurrences in occurrence tree can be mapped to STEP using action and action_relationship.

Fluents can be mapped to "State_type" and through the "applied_state_type_assignment" to an action or an action_resource entity. State_relationship can be represented using state_relationship.

Fluents can also be used to determine the conditions under which an activity must occur. This is different from preconditions which only specify the conditions under which an activity may possibly occur. The latter is synonymous to Events in DES therefore these fluents are mapped to STEP using the "Event_occurrence" entity. Event_occurrence is assigned to an action using applied_event_occurrence_assignment.

Figure 3 illustrates a manufacturing cell, including four operations, Trim, Test, Paint and Assemble. For the purpose of balancing it has been decided that the number of work pieces in the cell must not exceed 10. Hence, there is a gate in the model which opens whenever the number of items becomes less than 10. There is a rework process which is initiated whenever a work piece is rejected by the test process. Figure 4 shows PSL representation of manufacturing processes of the cell. The definition of objects, input and outputs of the processes activity is omitted for simplicity and only process occurrences, and their corresponding conditions are represented. Figure 5 represents the instantiated schema of the cell in STEP 239 according to the PSL model.

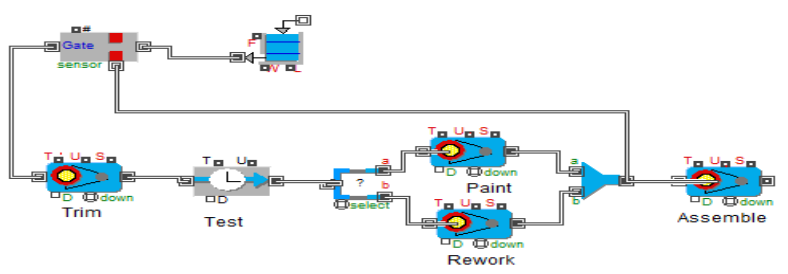

Figure 3. An example of a manufacturing cell

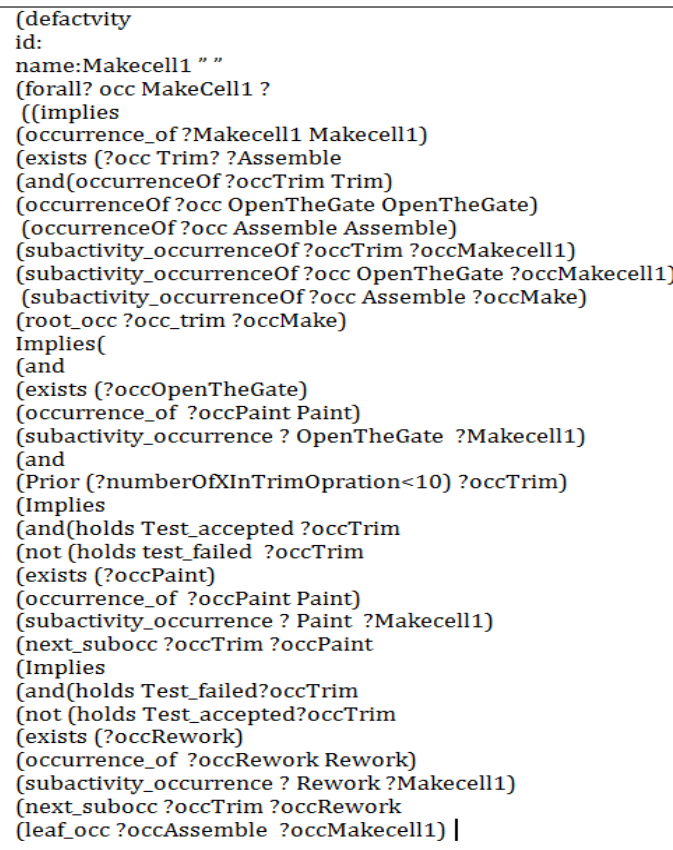

Figure 4.PSL representation of manufacturing processes of the cell 
In figure 5 \# 1 represents the aggregated manufacturing processes in the cell.There are 6 "Action_relationships" that connect Action \#1 with its subactivities actions \#6, 7, 8, 9, 11 and 20.The "Name" property of these entities are according to the definitions and concepts in PSL representation (see figure 4).Then these entities connect the Action \# 1 to the related manufacturing processes (actions in PLCS).For instance; Entity "Action_relationship" \#4 represents that the action \# 8 , Trim process, is the root occurrence of this cell.This action has relationship with Entity "Action" \# 7 (Test) and the type of relationship is "next_subocc".This action has two relationships with action \#11(Paint) and action \#9 (Rework) through "Action_relationships"\# 17 and \#13 respectively. "Action_relationship" \#13 is connected with "Applied_state_type_assignment" which shows the relationship of "Test" action and "Rework "action is conditional which in this case is the result of the "Test" action.Hence if the ressult of test is fail then the "Rework" action must be executed.Entity \# 15 "State_type" is the state assigned to the "applied_stae_type_assignment" whose name property is "test failed" and entity \# 16 determines the role of entity "applied_stae_type_assignment".As aformentioned in the cell description, the number of products in this cell must not be greater than 10. "Action \# 20" is represents the activity of letting the products in the cell whenever the number of products in the cell is less than 10.Entities \# 21 "Applied_event_occurrence_assignment" , \# 22 "event_occurrence" and \# 23 „Event_occurrence_role“" represent the event for this purpose.Entity \#22 "Event_occurrence“ defines the event ; whenever a product leaves the cell or whenever the number of produts in the cell is less than 10.This entity is connected to "Action \#20" "Open the gate"through the entity \#24 "Applied_action_assignment".Entity \#11"Action:Paint" has a relationship with Entity \#6 "Action: Assemble" through the "Action_relationship" entity \#18.

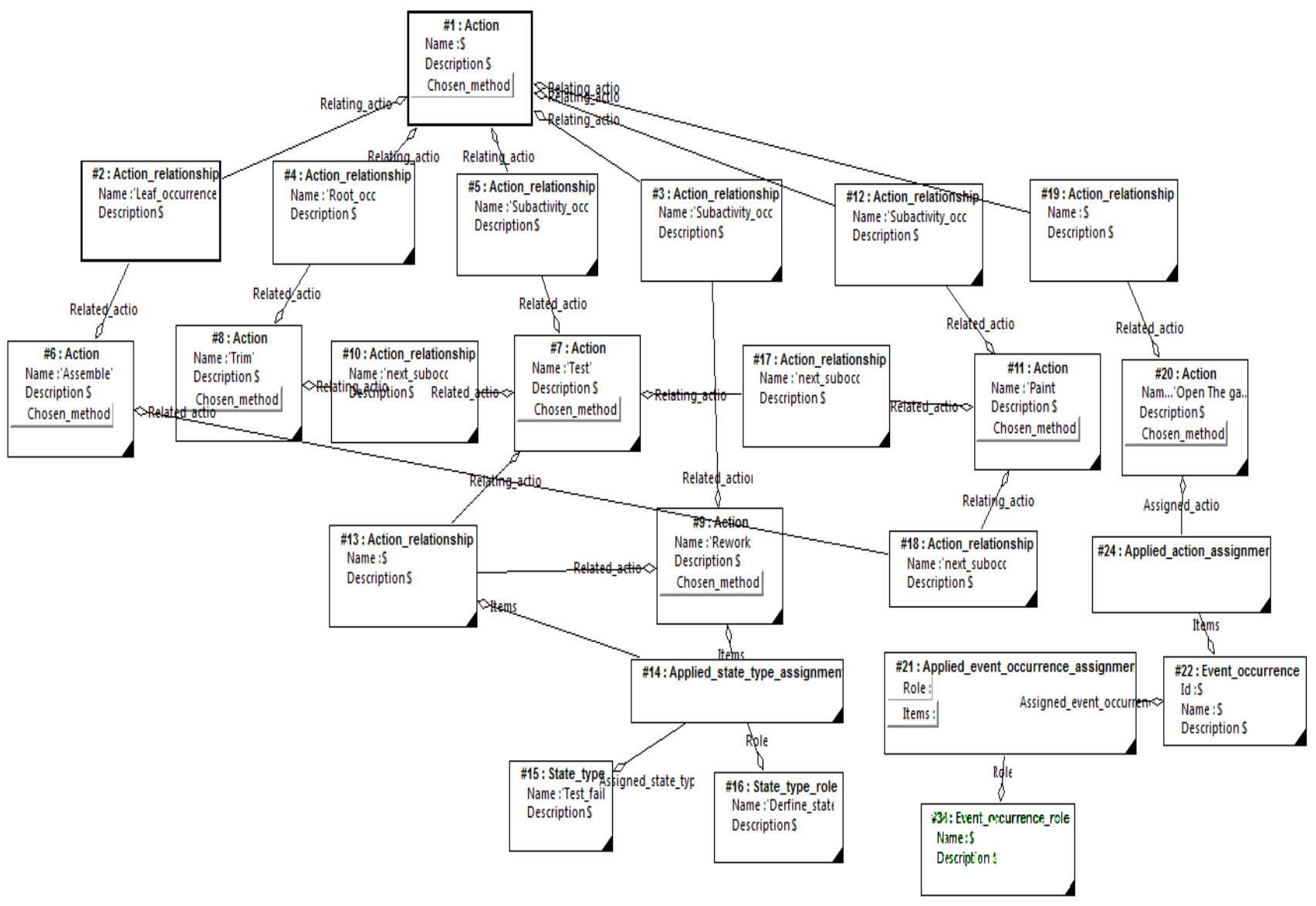

Figure 5.Instantiated schema of the cell in STEP 239 according to the PSL model 


\section{Conclusion}

A fundamental requirement for implementing Discrete Event Simulation (DES) requires representing operation sequences while considering their necessary resources and inputs and outputs as well as a detailed description of specifications and constraints on processes occurrences. The PLCS standard can represent desired information including activity, activity relationship, states, events, resource, product and their interrelationship.However; PLCS is a generic information standard without the semantics of a domain and need guidance for consistent representation of aforementioned information in DES modelling. The PSL ontology introduces a way to disambiguate manufacturing process modelling constructs, their sequence and behaviour execution by expressing their semantics as constraints on runtime sequences of manufacturing processes behaviour. However it is specific for the process specification domain which requires a definition of how to integrate with other domains of information. Such related domains include geometry, the stochastic representation of manufacturing processes properties or versioning and change management. Therefore this paper presents an approach to use the PSL ontology as a reference for a manufacturing process specification, mapping it to the STEP standard. This approach increases the level of semantics and level of interoperability among users and systems and makes the integration of manufacturing processes specification with other data more convenient.

\section{References}

[1] V.E.Chelpin, "Information modelling for the manufacturing system life cycle", Doctoral thesis, Royal Institute of Technology. Stockholm,. Sweden, 2008.

[2] P. Falkman, J.Nielsen, B. lennartson, A. Cheplin,"Generation of STEP AP214 Models From Discrete Event Systems for Process Planning and Control", IEEE transaction On Automation Science and Engineering, Vol. 5, NO. 1, 2008.

[3] M. Gruninger, "Ontology of the Process Specification Language", Institute for Systems Research, University of Maryland, College Park, 2001.

[4] ISO 10303-239 (2005), ISO/TC184/SC4 : ISO 10303-239 : Industrial automation systems and integration - Product Data Representation and Exchange - Part 239: Application protocol: Product Life Cycle Support, First Edition, 2005.1

[5] M. Gruninger, "Ontology of the Process Specification Language", Institute for Systems Research, University of Maryland, College Park, 2001. 